\title{
Magnetic resonance imaging-based synthetic computed tomography of the lumbar spine for surgical planning: a clinical proof-of-concept
}

\author{
Victor E. Staartjes, BMed, ${ }^{1-3}$ Peter R. Seevinck, PhD, ${ }^{4,5}$ W. Peter Vandertop, MD, PhD, ${ }^{2}$ \\ Marijn van Stralen, PhD, ${ }^{4,5}$ and Marc L. Schröder, MD, PhD'1
}

1Department of Neurosurgery, Bergman Clinics, Amsterdam; ${ }^{2}$ Amsterdam UMC, Vrije Universiteit Amsterdam, Neurosurgery, Amsterdam Movement Sciences, Amsterdam; ${ }^{4}$ Image Sciences Institute, University Medical Center Utrecht; and ${ }^{5}$ MRIguidance B.V., Utrecht, The Netherlands; and ${ }^{3}$ Machine Intelligence in Clinical Neuroscience (MICN) Laboratory, Department of Neurosurgery, University Hospital Zurich, Clinical Neuroscience Centre, University of Zurich, Switzerland

OBJECTIVE Computed tomography scanning of the lumbar spine incurs a radiation dose ranging from $3.5 \mathrm{mSv}$ to $19.5 \mathrm{mSv}$ as well as relevant costs and is commonly necessary for spinal neuronavigation. Mitigation of the need for treatment-planning CT scans in the presence of MRI facilitated by MRI-based synthetic CT (sCT) would revolutionize navigated lumbar spine surgery. The authors aim to demonstrate, as a proof of concept, the capability of deep learningbased generation of SCT scans from MRI of the lumbar spine in 3 cases and to evaluate the potential of sCT for surgical planning.

METHODS Synthetic CT reconstructions were made using a prototype version of the "BoneMRl" software. This deep learning-based image synthesis method relies on a convolutional neural network trained on paired MRI-CT data. A specific but generally available 4-minute 3D radiofrequency-spoiled T1-weighted multiple gradient echo MRI sequence was supplemented to a 1.5T lumbar spine MRI acquisition protocol.

RESULTS In the 3 presented cases, the prototype SCT method allowed voxel-wise radiodensity estimation from MRI, resulting in qualitatively adequate CT images of the lumbar spine based on visual inspection. Normal as well as pathological structures were reliably visualized. In the first case, in which a spiral CT scan was available as a control, a volume CT dose index $\left(\mathrm{CTDI}_{\mathrm{vol}}\right)$ of $12.9 \mathrm{mGy}$ could thus have been avoided. Pedicle screw trajectories and screw thickness were estimable based on SCT findings.

CONCLUSIONS The evaluated prototype BoneMRI method enables generation of SCT scans from MRI images with only minor changes in the acquisition protocol, with a potential to reduce workflow complexity, radiation exposure, and costs. The quality of the generated CT scans was adequate based on visual inspection and could potentially be used for surgical planning, intraoperative neuronavigation, or for diagnostic purposes in an adjunctive manner.

https://thejns.org/doi/abs/10.3171/2020.10.FOCUS20801

KEYWORDS lumbar spine; image conversion; imaging; deep learning; machine learning; artificial intelligence

I N complex lumbar spinal conditions such as spondylolisthesis, kyphoscoliosis, or spinal tumors, the combination of MRI and CT have long proven complementary. Whereas CT imaging perfectly visualizes osseous structures and allows for assessment of spinal integrity and stability, MRI excels at delineating soft-tissue and nervous system structures with high contrast. CT images are often required for neuronavigation and treatment planning, such as for image-guided navigated biopsies or pedicle screw placement. ${ }^{1}$

However, CT scanning is inherently coupled to radia- tion exposure; an average lumbar spinal CT study equals an effective dose of around $3.5 \mathrm{mSv}$ up to $19.5 \mathrm{mSv}^{2-6}$ In addition, multiple imaging sessions increase the patient burden and overall costs and introduce complex workflows, with the potential for intermodality registration errors. For these reasons, MRI-only workflows have gained attention in recent years. ${ }^{7,8}$

In this brief report, we evaluate a prototype of the "BoneMRI" method, for the first time applied to the lumbar spine, that allows generation of synthetic CT (sCT) images from generally available MRI based on deep learn- 

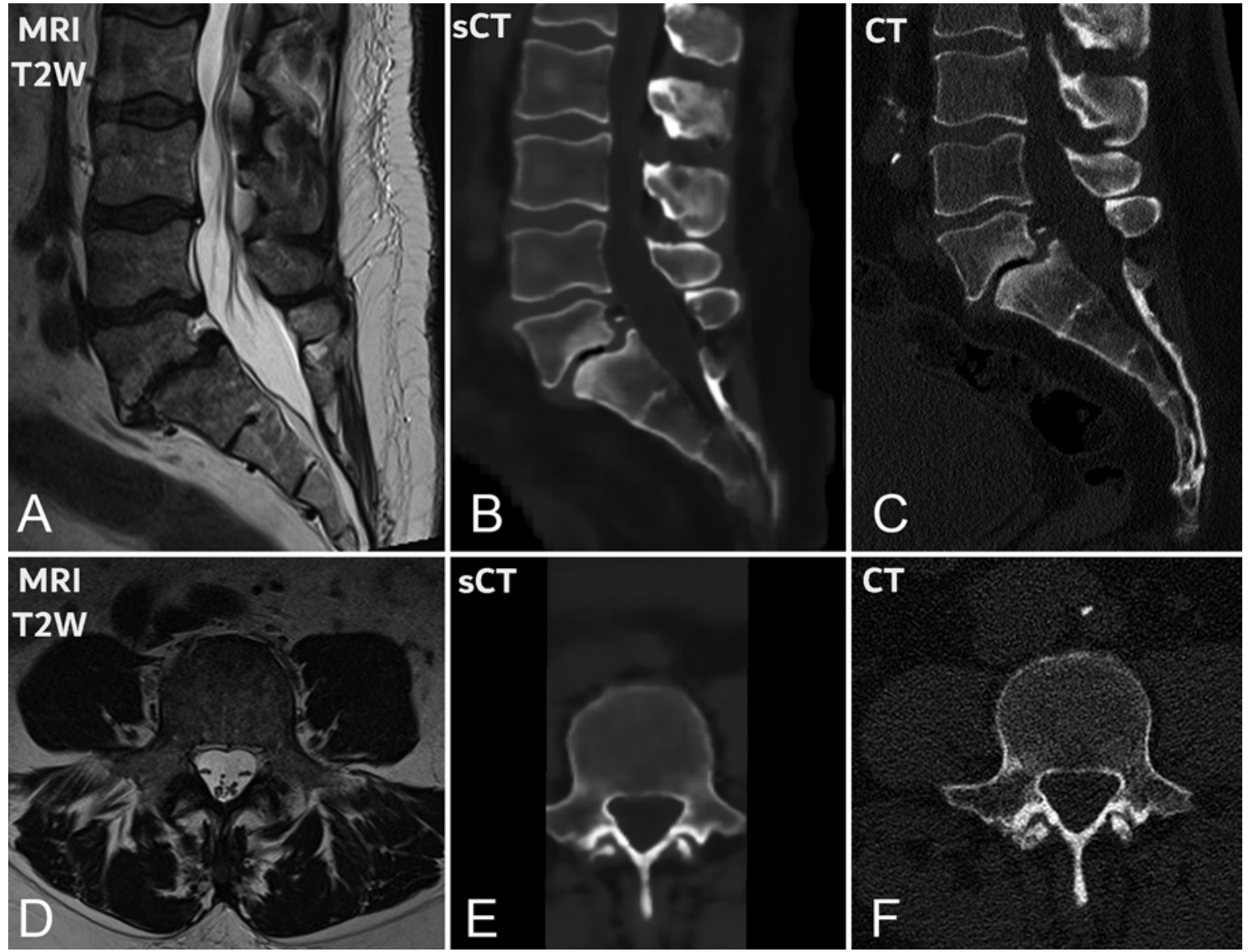

FIG. 1. Case 1 (test data set). A and D: Conventional T2W MR images acquired on a 1.5T scanner. B, E, C, and F: Synthetic CT images generated from the BoneMRI sequence ( $B$ and $E$ ), along with the ground truth spiral CT scans ( $C$ and $F)$. Midsagittal and axial (L5 pedicles) cuts are displayed in the upper and lower panels, respectively. This patient's BoneMRI sequence was acquired with a field of view width of $7.2 \mathrm{~cm}$, with the transverse processes being cut off consequently.

ing. In this proof-of-concept study, we hypothesized that MRI-based sCT images can be used for surgical planning, potentially rendering planning CT scans superfluous.

\section{Methods \\ Overview}

We utilized a research prototype of the BoneMRI sCT generation method (BoneMRI, MRIguidance B.V.), which is based on preliminary work. ${ }^{9-11}$ The deep learning model was trained using paired MRI and CT data, partly obtained in the context of this study and partly in other studies. ${ }^{9-11}$ We included 9 patients who were scheduled for robotic lumbar fusion surgery, of whom 8 were used in the training set, independent from the test set. The test set consisted of one of the 9 included patients scheduled for lumbar fusion surgery and 2 volunteers.

\section{Image Acquisition}

$\mathrm{CT}$ images were acquired in the supine position using a Philips ICT 256 scanner (318 slices, 1-mm thickness) and a lumbar spine protocol with iDose reconstruction. MR images were acquired in a fixed supine position using a Siemens Magnetom Essenza (1.5T field strength).
A standard lumbar spine protocol including conventional T1-weighted (T1W) and T2-weighted (T2W) sequences (acquisition time 15 minutes and 4 seconds), was complemented by a sagittal 3D radiofrequency-spoiled T1W multiple gradient echo) sequence for BoneMRI reconstruction (2 echoes; TR 7 msec, TE1 $2.1 \mathrm{msec}$, TE2 $4.2 \mathrm{msec}$; FOV $250 \times 250 \times 90 \mathrm{~mm}$; reconstructed voxel size $0.74 \times 0.74$ $\times 0.9 \mathrm{~mm}$, acquisition time 3 minutes and 53 seconds). This dedicated sequence utilized a high-frequency encode bandwidth (BW > $500 \mathrm{~Hz} /$ pix) to minimize potential geometrical distortions.

\section{Model Development}

Synthetic CT scans were generated from MRI inputs using a patch-based convolutional neural network, similar to U-Net, with CT scans as the ground truth. ${ }^{9,12}$ The model inputs consisted of 4D MRI scans with 3 spatial dimensions and 1 channel dimension. ${ }^{9}$ The network was implemented in Keras ${ }^{13}$ with a TensorFlow backend (Google Brain Team, Google LLC).

\section{Proof-of-Concept Study}

The prototype algorithm was applied to 3 subjects: 1 

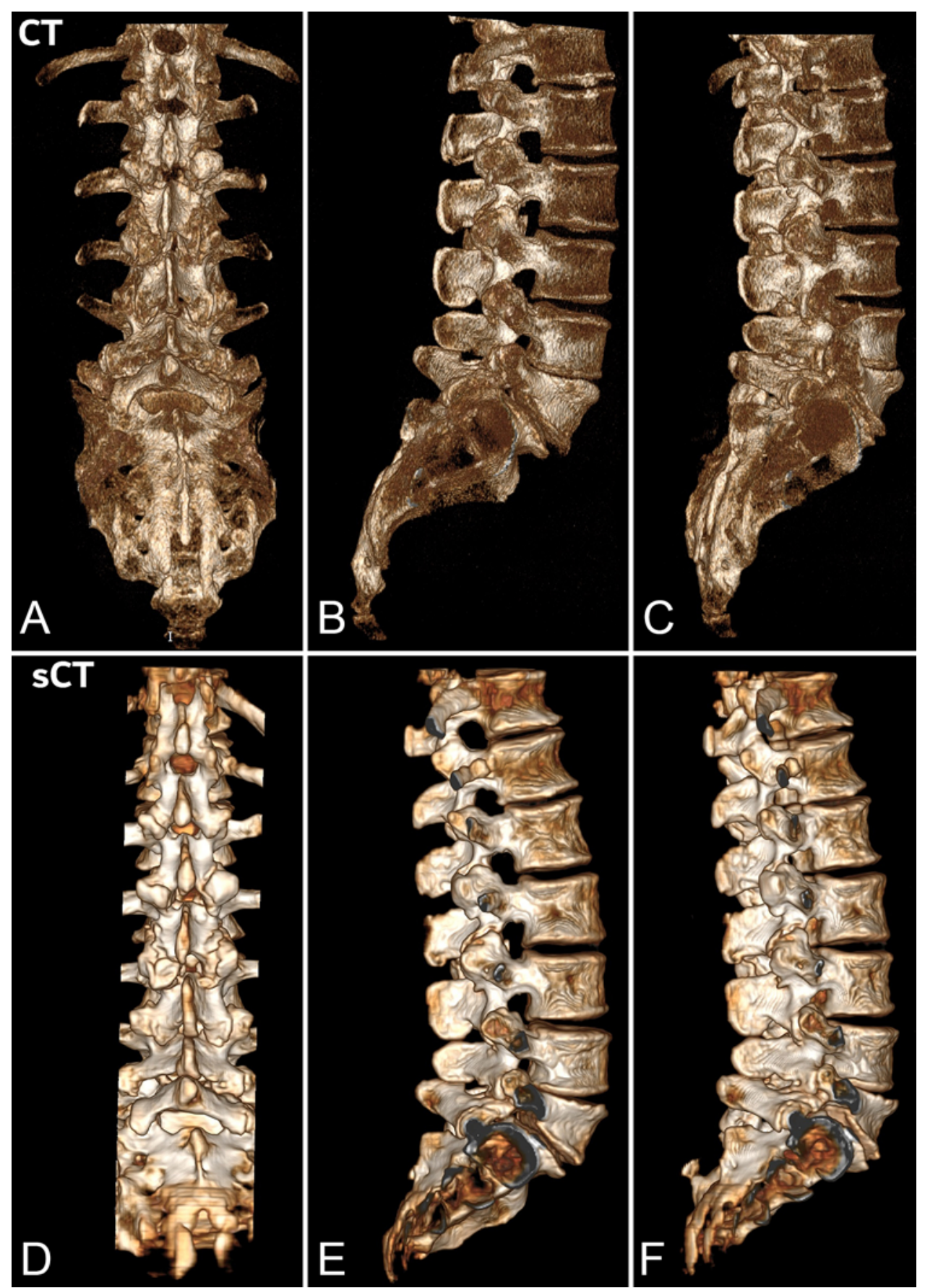

FIG. 2. Case 1 (test data set). Comparison of $3 \mathrm{D}$ volume renderings of the lumbar spine calculated from conventional $\mathrm{CT}(\mathrm{A}-\mathrm{C})$ and from an SCT generated from the BoneMRI sequence in the same patient (D-F). Posterior (A and D), lateral (B and E), and oblique ( $C$ and $F$ ) views are provided. This patient's BoneMRI sequence was acquired with a field of view width of $7.2 \mathrm{~cm}$, with the transverse processes being cut off consequently.

patient scheduled for lumbar fusion surgery (case 1) and 2 healthy volunteers (cases 2 and 3 ). In case 1, conventional T1W and T2W MRI sequences as well as the BoneMRI sequence and the generated $\mathrm{sCT}$ scans were available, along with a conventional CT scan of the lumbar spine. For cases 2 and 3, we tested the algorithm in its intended use case; only a BoneMRI sequence of the lumbar spine was available, and from it an sCT scan was generated. These 3 individuals were never before encountered by the algorithm, thus representing a valid test object. Multiplanar reconstructions and 3D volume renderings were generated in RadiAnt Version 2020.1, and manual as well as semiautomated measurements and pedicle screw trajectory planning were carried out in Surgimap Version 2.3.2.1. 
TABLE 1. Exemplary measurements performed comparatively on synthetic $C T$ and spiral CT in case 1

\begin{tabular}{lccc}
\hline \multicolumn{1}{c}{ Measurement } & sCT & Spiral CT & Difference \\
\hline L3 & & & \\
\hline Anterior VBH & 26.5 & 26.5 & 0.0 \\
\hline Posterior VBH & 32.0 & 31.8 & 0.2 \\
\hline Spinal canal diameter & 14.9 & 15.0 & -0.1 \\
\hline L4 & & & \\
\hline Anterior VBH & 27.2 & 26.8 & 0.4 \\
\hline Posterior VBH & 29.3 & 28.9 & 0.4 \\
\hline Spinal canal diameter & 19.1 & 18.9 & 0.2 \\
\hline L5 & & & \\
\hline Anterior VBH & 29.1 & 29.1 & 0.0 \\
\hline Posterior VBH & 11.8 & 12.0 & -0.2 \\
\hline Spinal canal diameter & 24.8 & 25.6 & -0.8 \\
\hline Total (MAD \pm SD) & & & $0.26 \pm 0.24$ \\
\hline MAD &
\end{tabular}

$M A D=$ mean absolute difference; $\mathrm{VBH}=$ vertebral body height.

Measurements are provided in millimeters.

\section{Ethical Considerations}

The development and proof-of-concept testing of the model and the associated use of patient data were approved by the local ethics review board (Medical Ethics Committees United). All patients signed informed consent forms that allow for the use of their data for research and publication purposes.

\section{Results}

Synthetic CT images of the lumbar spines of all 3 cases were successfully generated from BoneMRI sequences. Based on visual inspection, the quality of the sCT scans was adequate.

Figure 1 illustrates a comparison of T2W MRI, spiral $\mathrm{CT}$, and sCT images in case 1 . In this case, a spiral CT scan was available as a control with a volume CT dose index of 12.9 mGy that could thus have been avoided. Three-dimensional volume renderings of the lumbar spine were also calculated from both spiral CT and SCT scans for comparison (Fig. 2). In addition, exemplary comparative measurements of anterior and posterior vertebral body height and spinal central canal diameter were performed in case 1 (Table 1), with a mean absolute difference of 0.26 $\pm 0.24 \mathrm{~mm}$.

To evaluate the proof-of-concept MRI-only surgical planning workflow, the algorithm was validated in $2 \mathrm{pa}$ tients who did not undergo CT scanning (cases 2 and 3). Figure 3 illustrates the BoneMRI sequence and the sCT scans generated from it for both test cases. Normal as well as pathological structures were reliably visualized (e.g., the relevant spondylolisthesis of one of the volunteers). Figure 4 illustrates that conventional measurements such as spinal canal diameter, lumbar lordosis, and spondylolisthesis grading, as well as semiautomated measurements such as vertebral body segmentation could be carried out reliably on sCT. In addition, we were able to plan pedicle screw trajectories and screw thicknesses based on sCT.

\section{Discussion}

We show that generation of SCT images of the lumbar spine from MRI is feasible. The ability to visualize the osseous structures in 3D in a similar fashion as traditionally done using CT imaging without radiation and without the need for a separate second examination will be useful in the neurosurgical treatment of spinal disorders, both for diagnostic and therapeutic purposes such as in neuronavigation.

The use of image translation algorithms in medicine has previously gained interest in other applications-notably concomitant with an increase of combined use of MRI and CT in the field of radiotherapy. ${ }^{14,15}$ For example, generation of sCT scans from MRI has been described for radiotherapy purposes in the head and neck, pelvis, prostate, torso, and brain, again mostly for radiotherapy planning. ${ }^{16-21} \mathrm{~A}$ variety of atlas-based or voxel-based methods have been described, using different input sequences, as summarized by Florkow et al. ${ }^{9}$ Concomitantly, improvements in image processing techniques such as statistical or machine learning models have also helped the field leap forward ${ }^{21}$ However, most applications have created substitute CT scans for radiation treatment planning purposes, often not achieving an image quality that would also be sufficient for diagnostic imaging or detailed neurosurgical planning. Apart from these aspects, generation of sCT scans of the lumbar spine has not previously been demonstrated, although preliminary work has been carried out focusing on the cervical spine. ${ }^{10,11}$

The BoneMRI technique evaluated in this brief report is a deep learning-based method that requires dedicated input data obtained using a generally available sagittal 3D radiofrequency-spoiled T1W multiple gradient sequences, with its parameters carefully chosen in order to sensitize for specific tissue properties. Due to the dual-echo approach, information about proton density, water and fat fractions, relaxation constants, and susceptibility was intrinsically provided to the deep learning model. ${ }^{9}$ Indeed, the results of the BoneMRI technique appear promising in providing high-fidelity sCT images from MR images, with relevant improvements in elimination of radiation exposure, total examination time, and overall logistic efficacy.

An increase in MRI-only workflows has been seen in recent years. ${ }^{7,8}$ However, this would mean losing the radiodensitometric information provided by the CT scan, which is problematic because spine surgeons often require CT images to evaluate osseous anatomy such as dysplastic or twisted pedicles, and because MRI-based neuronavigation is still poorly implemented. In spinal neurosurgery, CT imaging has particular importance in surgical navigation, for example, for the computerassisted insertion of pedicle screws. ${ }^{22}$ Thus, high-fidelity sCT imaging combined with navigation systems or surgical robotics ${ }^{22-25}$ could enable the concept of radiationless navigated surgery, enabling the use of computer assistance based on preoperative CT imaging without the need for additional radiation. Still, intraoperative fluoroscopy may be necessary for registration and instrumentation control, but these fluoroscopic doses are minor compared with those experienced by the patient during CT scanning. ${ }^{2,3,26,27}$ Of course, intraoperative CT imag- 


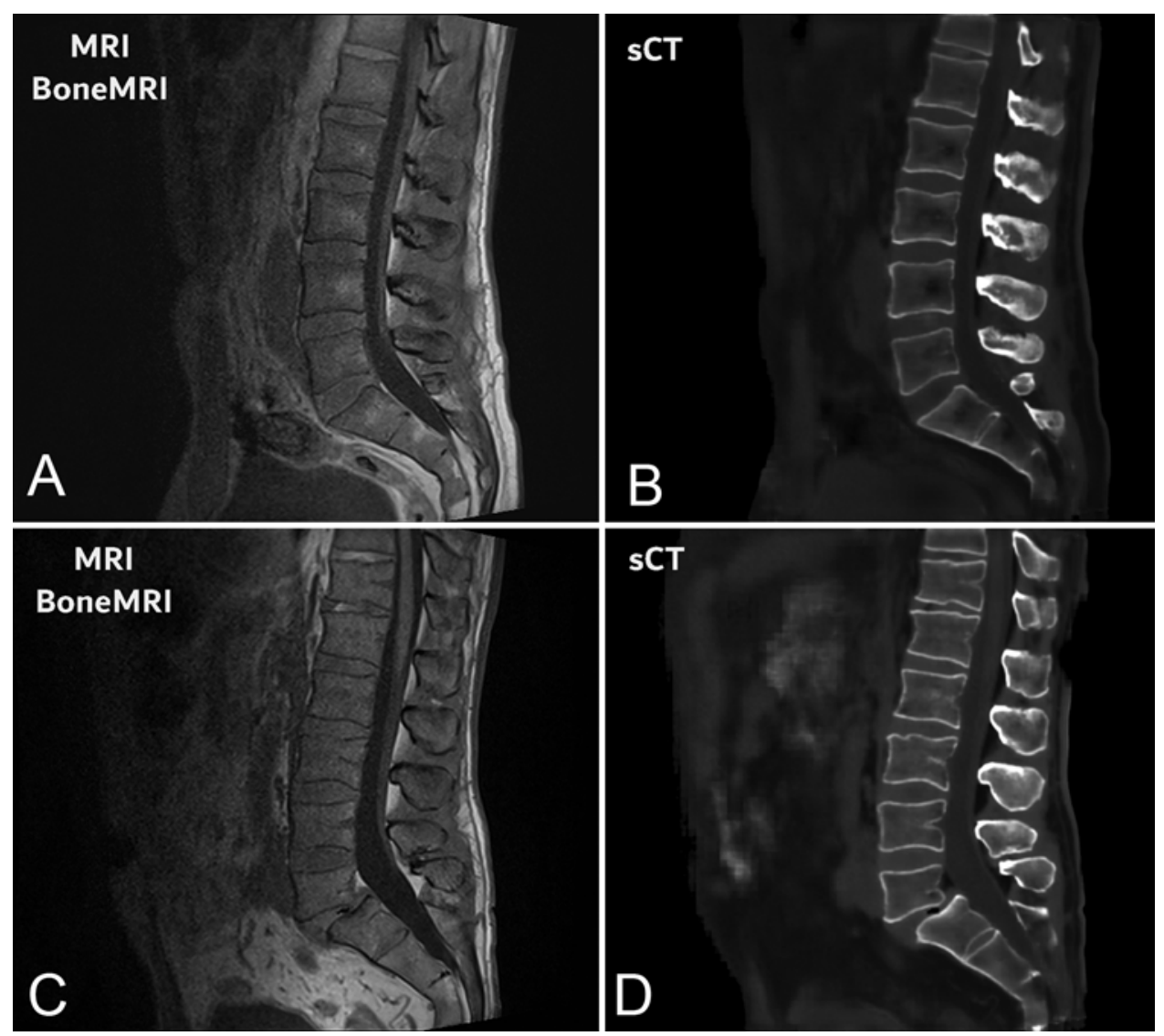

FIG. 3. BoneMRI sequences acquired in 2 volunteers (cases 2 [upper] and 3 [lower]) not represented in the training data set (A and $\mathrm{C}$ ), along with the corresponding SCT images generated (B and D). Midsagittal cuts of the lumbar spine are shown.

ing can also be particularly relevant in spine surgery, for acquisition of images for spinal neuronavigation based on the actual position of the patient on the operating table. Even under these circumstances, one could imagine the use of intraoperative MRI with generation of sCT scans instead of intraoperative CT, especially when considering the increased adoption of intraoperative MRI in neurosurgical departments, ${ }^{28,29}$ and the possibility for rapid MRI sequences..$^{30}$ In the near future, it is more likely that clinical applications of sCT scanning will focus around preoperative surgical planning for complex cases, implant sizing, and radiationless intraoperative navigation.

\section{Limitations}

The evaluated prototype algorithm is validated on limited data. Thus, although we show the results applied to 3 cases previously unseen by the algorithm, it has not yet been validated in patients with, for example, highly dysplastic or scoliotic pedicles, bone metastases, Modic-type endplate changes, and so forth. The limited amount of data prevents us from performing a thorough statistical analysis of geometricaccuracy. However, we applied a high-frequency encode bandwidth to minimize potential geometrical distortion. In addition, previous work has demonstrated the robustness of $\mathrm{sCT}$ generation to specific degenerative spine diseases, also confirming geometric accuracy of sCT in comparison with spiral CT. ${ }^{10,11}$ Furthermore, validation in patients with implants such as pedicle screws and rods, intervertebral cages, artificial intervertebral discs, neurostimulators, and interspinous process devices is required. Further development and validation on more patients are thus warranted, and the quality of the image might improve further with extended training. Similarly, to demonstrate the feasibility of using the generated sCT scans for accurate, near-radiationless robotic spine surgery, a case series is currently underway for clinical validation.

\section{Conclusions}

We show for the first time in the lumbar spine that, through the use of the BoneMRI acquisition sequence and convolutional neural networks, generation of SCT scans from MR images is feasible within minutes and with visually adequate $\mathrm{SCT}$ image fidelity. This novel method has the potential to reduce workflow complexity, radiation exposure, and costs associated with adjunctive CT scanning in the lumbar spine. The quality of the generated $\mathrm{SCT}$ scans-based on visual inspection-is sufficient for surgical planning and neuronavigation and may even suffice for diagnostics. Further validation of the method is warranted in patients with implants and other artifacts. Likewise, further development of the algorithm based on larger pa- 

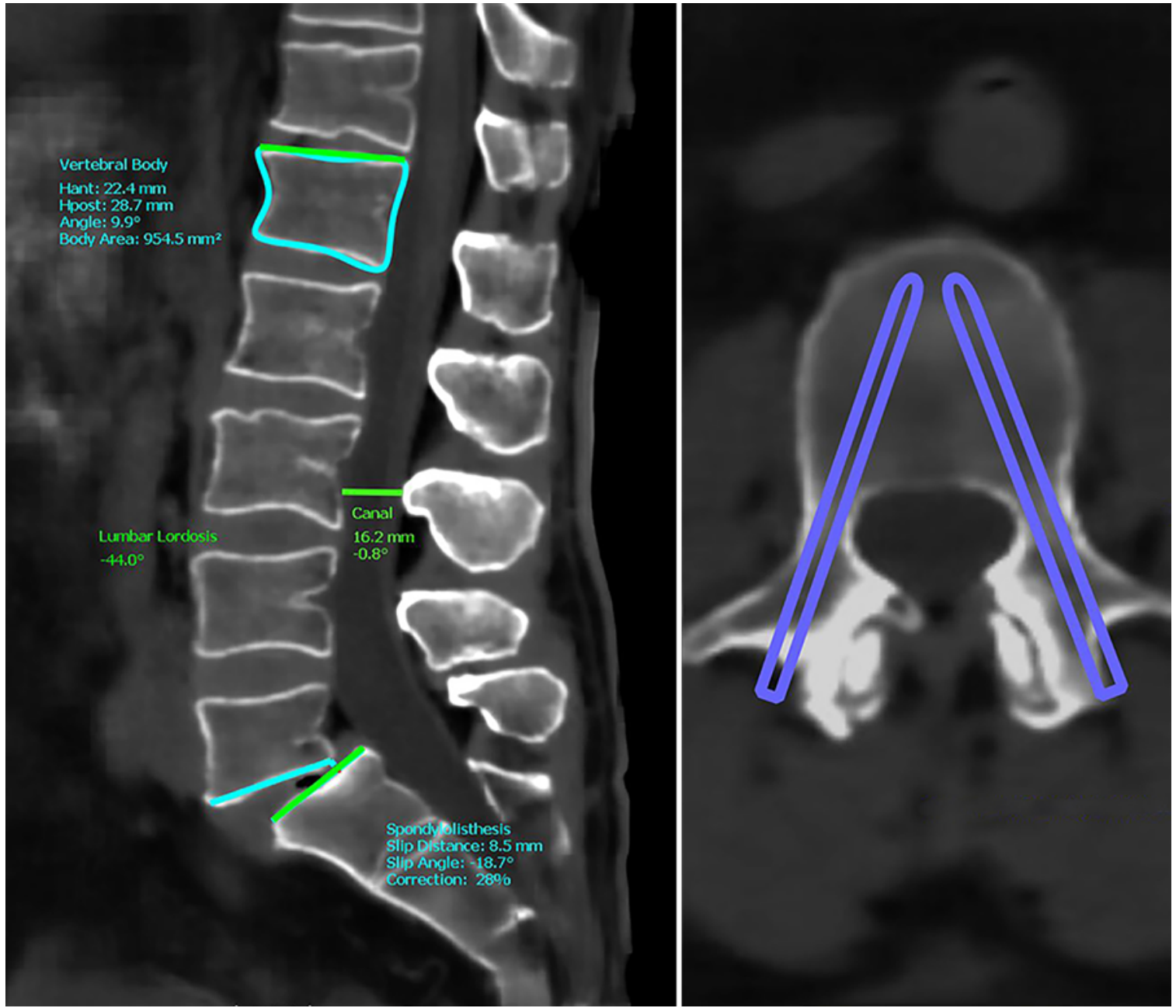

FIG. 4. Case 3. A midsagittal synthetic CT cut (left) in case 3 along with an axial reconstruction (right). Conventional measurements such as spinal canal diameter, lumbar lordosis, and spondylolisthesis grading (Meyerding grade II) as well as semiautomated measurements such as vertebral body segmentation were carried out. In addition, pedicle screw trajectories and screw thickness for both $\mathrm{L} 5$ pedicles were estimated on SCT imaging.

tient cohorts will likely improve image fidelity and consequently allow early clinical studies focusing on evaluating utility in surgical planning and intraoperative neuronavigation, as well as eventually radiationless robotic pedicle screw insertion.

\section{Acknowledgments}

We thank our radiation technologists Mirjam Visscher and Hester Knottnerus for their kind professional assistance.

\section{References}

1. Härtl R, Lam KS, Wang J, et al. Worldwide survey on the use of navigation in spine surgery. World Neurosurg. 2013;79(1): 162-172.

2. Bohl DD, Hijji FY, Massel DH, et al. Patient knowledge regarding radiation exposure from spinal imaging. Spine $J$. 2017;17(3):305-312.

3. Biswas D, Bible JE, Bohan M, et al. Radiation exposure from musculoskeletal computerized tomographic scans. J Bone Joint Surg Am. 2009;91(8):1882-1889.
4. Lin EC. Radiation risk from medical imaging. Mayo Clin Proc. 2010;85(12):1142-1146.

5. Papachristodoulou A, Pliamis N, Volford G, et al. Radiation dose of lumbar spine CT: analysis and comparison between different modes of acquisition in two European imaging centers. Poster presented at: European Congress of Radiology 2016; March 2-6, 2016; Vienna, Austria. Accessed November 18, 2020. https://epos.myesr.org/poster/esr/ecr2016/C-2386

6. Richards PJ, George J, Metelko M, Brown M. Spine computed tomography doses and cancer induction. Spine (Phila Pa 1976). 2010;35(4):430-433.

7. De Silva T, Uneri A, Ketcha MD, et al. Registration of MRI to intraoperative radiographs for target localization in spinal interventions. Phys Med Biol. 2017;62(2):684-701.

8. Owrangi AM, Greer PB, Glide-Hurst CK. MRI-only treatment planning: benefits and challenges. Phys Med Biol. 2018; 63(5):05TR01.

9. Florkow MC, Zijlstra F, Willemsen K, et al. Deep learningbased MR-to-CT synthesis: the influence of varying gradient echo-based MR images as input channels. Magn Reson Med. 2020;83(4):1429-1441.

10. van der Kolk BYM, van Stralen M, Podlogar M, et al. Re- 
construction of osseous structures in MRI scans of the cervical spine with BoneMRI: a quantitative analysis. Poster presented at: ASNR 57th Annual Meeting; May 18-23, 2019; Boston, MA.

11. van Stralen M, Podlogar M, Hendrikse J, et al. BoneMRI of the cervical spine: deep learning-based radiodensity contrast generation for selective visualization of osseous structures. Paper presented at: ISMRM 27th Annual Meeting and Exhibition, May 11-16, 2019; Montreal, QC, Canada.

12. Cui Z, Yang J, Qiao Y. Brain MRI segmentation with patchbased CNN approach. In: 2016 35th Chinese Control Conference (CCC). IEEE; 2016:7026-7031.

13. Chollet F. Keras: Deep learning library for Theano and TensorFlow. Accessed Dec 8, 2020. https://keras.io

14. Pollard JM, Wen Z, Sadagopan R, et al. The future of imageguided radiotherapy will be MR guided. Br J Radiol. 2017; 90(1073):20160667.

15. Dirix P, Haustermans K, Vandecaveye V. The value of magnetic resonance imaging for radiotherapy planning. Semin Radiat Oncol. 2014;24(3):151-159.

16. Edmund JM, Nyholm T. A review of substitute CT generation for MRI-only radiation therapy. Radiat Oncol. 2017;12(1):28.

17. Maspero M, Savenije MHF, Dinkla AM, et al. Dose evaluation of fast synthetic-CT generation using a generative adversarial network for general pelvis MR-only radiotherapy. Phys Med Biol. 2018;63(18):185001.

18. Dinkla AM, Wolterink JM, Maspero M, et al. MR-only brain radiation therapy: dosimetric evaluation of synthetic CTs generated by a dilated convolutional neural network. Int $J$ Radiat Oncol Biol Phys. 2018;102(4):801-812.

19. Dinkla AM, Florkow MC, Maspero M, et al. Dosimetric evaluation of synthetic CT for head and neck radiotherapy generated by a patch-based three-dimensional convolutional neural network. Med Phys. 2019;46(9):4095-4104.

20. Siversson C, Nordström F, Nilsson T, et al. Technical Note: MRI only prostate radiotherapy planning using the statistical decomposition algorithm. Med Phys. 2015;42(10):6090-6097.

21. Edmund JM, Kjer HM, Van Leemput K, et al. A voxel-based investigation for MRI-only radiotherapy of the brain using ultra short echo times. Phys Med Biol. 2014;59(23):75017519.

22. Staartjes VE, Klukowska AM, Schröder ML. Pedicle screw revision in robot-guided, navigated, and freehand thoracolumbar instrumentation: a systematic review and meta-analysis. World Neurosurg. 2018;116:433-443.e8.

23. Schröder ML, Staartjes VE. Revisions for screw malposition and clinical outcomes after robot-guided lumbar fusion for spondylolisthesis. Neurosurg Focus. 2017;42(5):E12.

24. Staartjes VE, Molliqaj G, van Kampen PM, et al. The European Robotic Spinal Instrumentation (EUROSPIN) study: protocol for a multicentre prospective observational study of pedicle screw revision surgery after robot-guided, navigated and freehand thoracolumbar spinal fusion. BMJ Open. 2019; 9(9):e030389.

25. Siccoli A, Klukowska AM, Schröder ML, Staartjes VE. A systematic review and meta-analysis of perioperative parameters in robot-guided, navigated, and freehand thoracolumbar pedicle screw instrumentation. World Neurosurg. 2019;127: 576-587.e5.

26. Mendelsohn D, Strelzow J, Dea N, et al. Patient and surgeon radiation exposure during spinal instrumentation using intraoperative computed tomography-based navigation. Spine $J$. 2016;16(3):343-354.

27. Villard J, Ryang YM, Demetriades AK, et al. Radiation exposure to the surgeon and the patient during posterior lumbar spinal instrumentation: a prospective randomized comparison of navigated versus non-navigated freehand techniques. Spine (Phila Pa 1976). 2014;39(13):1004-1009.

28. Stienen MN, Fierstra J, Pangalu A, et al. The Zurich Checklist for safety in the intraoperative magnetic resonance imaging suite: technical note. Oper Neurosurg (Hagerstown). 2019;16(6):756-765.

29. Staartjes VE, Serra C, Maldaner N, et al. The Zurich Pituitary Score predicts utility of intraoperative high-field magnetic resonance imaging in transsphenoidal pituitary adenoma surgery. Acta Neurochir (Wien). 2019;161(10):2107-2115.

30. Sayah A, Jay AK, Toaff JS, et al. Effectiveness of a rapid lumbar spine MRI protocol using 3D T2-weighted SPACE imaging versus a standard protocol for evaluation of degenerative changes of the lumbar spine. AJR Am J Roentgenol. 2016;207(3):614-620.

\section{Disclosures}

M.v.S. and P.R.S. are cofounders and co-owners of MRIguidance B.V.

\section{Author Contributions}

Conception and design: Staartjes, Seevinck, van Stralen, Schröder. Acquisition of data: Seevinck, van Stralen, Schröder. Analysis and interpretation of data: Staartjes, Seevinck, van Stralen, Schröder. Drafting the article: Staartjes. Critically revising the article: Seevinck, Vandertop, van Stralen, Schröder. Reviewed submitted version of manuscript: all authors. Approved the final version of the manuscript on behalf of all authors: Staartjes. Statistical analysis: Seevinck, van Stralen. Administrative/technical/material support: Seevinck, van Stralen, Schröder. Study supervision: Seevinck, Schröder.

\section{Correspondence}

Victor E. Staartjes: c/o Bergman Clinics, Naarden, The Netherlands.victor.staartjes@gmail.com. 